\section{La aerotermia como alternativa para el control de Triatoma infestans (Hemiptera, Reduviidae) resistentes a deltametrina}

\author{
Air temperature elevation as an alternative \\ for the control of deltamethrin-resistant \\ Triatoma infestans (Hemiptera, Reduviidae)
}

Alberto G. Gentile 1

José L. Sartini 2

María C. Campos 3

Juan F. Sánchez 4
1 Coordinación de Gestión Epidemiológica,

Ministerio de Salud Pública, Salta, Argentina.

2 Coordinación de Gestión de Riesgo Sanitario,

Zoonosis y Medio Ambiente,

Ministerio de Salud Pública

Salta, Argentina.

3 Vigilancia Epidemiológica,

Ministerio de Salud Pública,

Salta, Argentina.

4 Supervisión Intermedia

de APS, Ministerio de Salud

Pública, Salta, Argentina.

Correspondencia

A. G. Gentile

Coordinación de Gestión

Epidemiológica,

Ministerio de Salud Pública.

Centro Cívico Grand Bourg

(4400), Salta, Argentina.

agentile@gobiernosalta.gov.ar

\section{Abstract}

This study explores the effects of artificial air temperature elevation on the embryonic, nymphal, and adult forms of deltamethrin-resistant Triatoma infestans. In the laboratory, complete offspring of $\mathrm{T}$. infestans were exposed to increasing air temperatures to determine the viability of eggs and survival of nymphs and adults; in the field, the experiment was conducted in a poor rural dwelling previously identified as infested with the vector. The laboratory phase showed $100 \%$ non-viability of eggs and 100\% mortality of nymphs and adults exposed to airtight temperatures from $45^{\circ} \mathrm{C}$ to $55^{\circ} \mathrm{C}$, while the field phase succeeded in reproducing the heat's ovicidal effect and negative conversion of entomological assays in the experimental dwelling, as conducted over the course of 72 hours at 30, 60, and 90 days from the baseline evaluation. Artificial elevation of airtight temperature on dwellings proved to be an acceptable, harmless, and technically simple control method.

Chagas Disease; Triatoma; Vector Control; Insecticide Resistance

\section{Introducción}

La comunicación hecha recientemente por el Centro de Investigaciones de Plagas e Insectos (CIPEIN) 1,2 acerca de que la persistente infestación por Triatoma infestans en viviendas rurales de "La Toma", Salvador Mazza, Provincia de Salta, Argentina, obedecía a resistencia triatomínea a Deltametrina, motivó la búsqueda de alternativas no convencionales de control, factibles de instrumentar mediante acciones de Atención Primaria de la Salud (APS).

Para este fin, se trabajó en "El Chorro", paraje incluido en el sector de "La Toma", que posee población rural dispersa y se encuentra ubicado al noroeste de la Ciudad de Salvador Mazza, limitando al norte con la República de Bolivia (suburbios de la ciudad de Yacuiba). Posee clima subtropical con estación seca, temperatura anual media de $26^{\circ} \mathrm{C}$, humedad relativa del ambiente anual media del $63 \%$ y pertenece al paisaje geográfico denominado Yunga.

Allí se encuentran localizadas 89 viviendas, dispersas en un radio aproximado de $4,5 \mathrm{~km}^{2}$, enclavadas entre claros dedicados a cultivos de maíz, mandioca y zapallos, principalmente.

Lo habitan 422 personas, en su mayoría aborígenes pertenecientes a etnias de raíz guaranítica (complejo Chiriguano-Chané), que residen en su mayoría en ranchos de paredes de barro amasado y caña, con techos de quincho o chapa de cartón y pisos de tierra, aunque al- 
gunas poseen paredes de ladrillos o adobe, en su mayoría sin revocar. Una de las características comunes de las viviendas de este lugar es que poseen un solo dormitorio con una superficie promedio de unos 9m². Entre 1987 y 1999 la infestación de viviendas en este paraje se mantuvo por debajo de $3 \% 3$. En febrero de 2003, alcanzó $83 \%$, pese a haberse completado dos ciclos de rociado con Deltametrina el año anterior.

En los últimos 5 años no se han notificado casos de Chagas Agudo entre sus pobladores 4. La prevalencia de infección chagásica allí en el año 2002 fue de 59\%, siendo en embarazadas del $41 \%$ y en niños menores de 5 años del 38\%. En la actualidad se está llevando a cabo un nuevo muestreo de la infección chagásica en esta área para descartar transmisión vectorial reciente.

Al respecto cabe señalar que al inicio del Programa Provincial de Chagas en 1981, el sector registró en ese entonces infestaciones superiores al $80 \%$, por lo que se le incluyó en la fase de ataque químico con Hexaclorociclohexano $(\mathrm{HCH})$, permanecieron bajo evaluación entomológica trimestral (a cargo de los Agentes Sanitarios del Programa de APS) y rociado sistemático de las viviendas reinfestadas hasta el presente. Infestación que se mantuvo con índices cercanos $1 \%$ entre 1990 y 1999, año a partir del cual comenzó gradualmente a elevarse (en 1985 se comenzó el tratamiento con piretroides, sustancias que se han utilizado hasta noviembre de 2002, mes en que se constató la ineficacia de la Deltametrina, a raíz de lo cual el Programa Nacional de Chagas decidió la reutilización de fosforados como paliativo, mientras se estudian nuevas alternativas de control).

A raíz de ello, se decidió reexplorar la Aerotermia (AT), método experimentado con éxito en 1984 en el Chaco Salteño, región fitogeográfica ubicada al este de la Provincia de Salta.

Este consiste en elevar la temperatura del aire del interior de las habitaciones infestadas por tratomíneos utilizando como fuente de calor brasas de carbón vegetal colocadas en el suelo, mediante lo cual se consigue reducir drásticamente la población de vinchucas debido a sus efectos ovicidas e imagocidas, más la eliminación mecánica de los insectos que se fugan hacia el exterior. Se basa en observaciones que muestran que, al igual que otros organismos vivos, los insectos son capaces de sobrevivir únicamente dentro de ciertos límites marcados por factores ambientales como la temperatura, la humedad relativa o el fotoperíodo. Dentro de este rango, estos factores in- fluyen a su vez sobre el nivel de respuesta de actividades tales como: la alimentación, la dispersión, la puesta o el desarrollo. De todos los factores ambientales, el que ejerce un efecto mayor sobre el desarrollo de los insectos es, probablemente, la temperatura. Ello es debido principalmente a su importante incidencia sobre los procesos bioquímicos, al ser organismos poiquilotermos, es decir, "de sangre fría" $5,6,7,8,9,10$.

Esta investigación estuvo respaldada mediante instrumento legal emitido por el Ministerio de Salud Pública de Salta y tuvo como objetivo explorar los efectos de la elevación artificial de la temperatura del aire sobre las formas embrionarias, ninfales y adultas de T. infestans resistentes a Deltametrina.

\section{Metodología}

La investigación de la eficacia de la AT se llevó a cabo entre los meses de enero y junio de 2003, en dos fases de experimentación: una de laboratorio y otra de campo.

\section{Experimentación de laboratorio}

De huevos y un grupo de vinchucas capturadas en el interior de una de las viviendas asignadas al estudio perteneciente al paraje "El Chorro", lugar aledaño a "La Toma”, que también resistieron a los dos últimos rociados con Deltametrina, se separaron por azar un lote de 50 individuos (10 por estadio) y 60 huevos fecundados, que luego se dividieron en dos, respetando la proporcionalidad por estadio y fueron colocados en cápsulas de Petri. Por azar también, uno de los grupos se expuso a calor y el otro se utilizó como control.

Para ello se utilizó una estufa de cultivo estándar donde se expuso, durante 10 minutos y a rangos de temperaturas que se incrementaron de a $5^{\circ} \mathrm{C}$, la progenie completa de $T$. infestans. La temperatura inicial se fijó en $35^{\circ} \mathrm{C}$ y la final en $55^{\circ} \mathrm{C}$. Durante el tiempo de espera hasta que la estufa alcanzara el rango siguiente, los especímenes permanecieron a temperatura ambiente.

\section{Experimentación en terreno}

Durante la experiencia de campo, dos viviendas se seleccionaron al azar de entre las evaluadas como infestadas durante la Ronda Sani- 
taria que fue efectuada por los Agentes Sanitarios del Programa de APS, finalizada en diciembre de 2002.

Los moradores fueron informados completamente sobre las características de procedimiento y aceptaron colaborar con la experiencia.

Una de ellas fue elegida para práctica del método y la otra se asignó al estudio propiamente dicho.

La elevación térmica de las habitaciones infestadas (dormitorios en ambos casos), se realizó siguiendo el protocolo original utilizado por A. G. Gentile en 1984, que utiliza un procedimiento cuyo principio consiste en alcanzar una temperatura del aire en el interior de la habitación colonizada superior a los $65^{\circ} \mathrm{C}$ y mantenerla durante al menos 2 horas, encendiendo carbón vegetal.

Siguiendo cada uno de los pasos, se realizó evaluación entomológica de 1hora/hombre en interior de cada vivienda, utilizando gancho entomológico y linterna, y como expurgante Tetrametrina al $0,2 \%$, procediéndose a señalizar los nidos de triatomíneos, para continuar luego con el cálculo de la cantidad de carbón a encender - a razón de $1 \mathrm{~kg} / \mathrm{m}^{3}$ - para luego proceder a la preparación de la habitación a tratar (la misma que se requiere para el tratamiento químico), revisando cuidadosamente la presencia de inflamables artificiales (encendedores a gas, queroseno, solventes, pinturas, etc.). Seguidamente se hermetizaron desde el exterior las grietas y espacios por donde pudiera fugarse el calor con cartones y papel grueso, se colocó el termómetro a 1,5m del suelo cercano a la puerta para facilitar su lectura durante el calentamiento, mientras que los huevos fecundados testigo fueron puestos en cajas de fósforos que se introdujeron en grietas de la mampostería, a igual altura que el termómetro; a continuación, se encendió el carbón en el centro de las habitaciones. Una vez transformado en brasas, se cerraron las ventanas y se extendieron éstas uniformemente cubriendo la totalidad del piso, cerrando por último la puerta. Una vez alcanzados los $65^{\circ} \mathrm{C}$, el procedimiento se prolongó por espacio de 2 horas.

Se efectuó una nueva evaluación entomológica según técnica. Estas se repitieron a las 24 horas, 45 y 90 días, respectivamente. Los huevos testigo se observaron por 45 días, a temperatura ambiente, en la propia localidad.

El peor resultado esperado para la primera fase, fue la supervivencia - a $55^{\circ} \mathrm{C}$ - de hasta un $10 \%$ de los embriones y ninfas I, de $20 \%$ en ninfas II a $\mathrm{V}$ y de $30 \%$ en adultos.

Para la fase de terreno, fue la supervivencia de hasta un $20 \%$ de los embriones de los huevos fecundados utilizados como control y el hallazgo de hasta 10 ninfas o adultos a los 90 días del procedimiento, en 1 hora/hombre de evaluación.

\section{Definición de variables}

Las variables fueron: alteraciones (lentitud de desplazamiento, reacción lenta a los estímulos mecánicos y lumínicos), volteo (ninfa o adulto vivo que no se desplaza y que no se apoya correctamente en sus patas), muerte (ninfa o adulto que no presenta movimientos espontáneos y que no responde a la estimulación mecánica ni lumínica), infestación intradomiciliaria (colonización por T. infestans en todos sus estadios en el interior de la vivienda, excluyendo las paredes exteriores), infestación peridomiciliaria (colonización por T. infestans en todos sus estadios en cualquier sitio del perímetro hasta $50 \mathrm{~m}$ que rodea a la vivienda, incluyendo las paredes exteriores).

Las variables dependientes en la primera fase fueron: inviabilidad larvaria y mortalidad. En la segunda fase fueron: infestación intradomiciliaria e inviabilidad larvaria.

\section{Resultados}

\section{Fase de laboratorio}

En el lote de 30 huevos expuesto a calor, no se registraron nacimientos luego de 45 días de observación, mientras que la tasa de nacimientos en el grupo control (de igual tamaño) fue del $100 \%$.

En cuanto a las ninfas, el $100 \%$ de las de estadio I murieron luego de la exposición a $45^{\circ} \mathrm{C}$, mientras que las restantes lo hicieron durante la exposición a $55^{\circ} \mathrm{C}$, al igual que los adultos. Tanto las ninfas mayores como los adultos presentaron excitación a partir de los $40^{\circ} \mathrm{C}$, conducta de fuga a los $45^{\circ} \mathrm{C}$ y señales de daño severo a los $50 \circ \mathrm{C}$ (parálisis de extremidades, despliegue de la probóscide y volteo).

\section{Fase de terreno}

Tanto en la vivienda de adiestramiento como en la seleccionada para la prueba, se alcanzaron temperaturas de hasta $80^{\circ} \mathrm{C}$.

La evaluación inicial de la vivienda de práctica mostró numerosos nidos de T. infestans en grietas de las paredes abocados hacia el interior, efectuándose varias capturas de adultos y ninfas de distintos estadios en una hora-hombre. Se contabilizó más de medio centenar de ninfas I y II habitando el polvillo del suelo 11 y debajo de objetos. La mayoría de los huevos hallados 
estaban vacíos. La población triatomínea fue estimada en alrededor de trescientos individuos.

Durante la evaluación post-tratamiento inmediata al calentamiento se logró la captura de un ejemplar adulto vivo debajo de un tronco depositado en el suelo. Ocho vinchucas adultas dañadas por calor (deformadas, alas rotas, etc.) fueron recogidas del suelo por la dueña de casa al día siguiente. Una ninfa IV fue encontrada viva en la pared, detrás de un terrón, en la evaluación de las 48 horas No se hallaron insectos en la evaluación de las 72 horas.

En la vivienda seleccionada para la experiencia, se detectó infestación triatomínea abundante localizada en el apoyo de las chapas del techo sobre las paredes, así como en ropas y muebles, lográndose visualizar ejemplares de todos los estadios en una hora-hombre; 20 huevos embrionados fueron detectados en el cajón de un ropero. Durante el procedimiento de calentamiento se logró una adherencia satisfactoria al protocolo.

La evaluación inmediata fue negativa, encontrándose abundante volteo en el piso de la habitación. Dos insectos adultos marcados, colocados en una grieta de unos $15 \mathrm{~cm}$ de profundidad, avenada únicamente hacia el interior, fueron hallados muertos y deformados (alas desplegadas, abdomen lobulado, etc.) en fondo de la misma al finalizar el procedimiento.

En la evaluación hecha a las 24 horas pudo capturarse una vinchuca adulta viva, sin daño aparente, sobre uno de los tirantes del techo.

En cuanto a los huevos, los que se extrajeron de entre la ropa y de un cajón del ropero durante el acondicionamiento de la vivienda previo al procedimiento, se separaron en dos lotes: uno con 12 unidades fue colocado sobre el rellano de una ventana. El otro, de 8 huevos, fue utilizado como control. En el lote expuesto no se registraron eclosiones durante los 45 días de observación, mientras que el control hubo nacimientos a partir del segundo día, hasta alcanzar una fecundidad del $100 \%$ (8/8) en ese lapso. Ambos lotes se conservaron en recipientes oscuros y aireados a temperatura ambiente (28,5C de media en esa época del año en Salvador Mazza).

Hasta la evaluación de los 90 días la vivienda permaneció negativa.

\section{Discusión}

Los hallazgos de laboratorio coinciden con lo observado por A. G. Gentile en 1984 y son reproducibles en cualquier laboratorio. En cuanto a la experiencia de campo, aquella fue reali- zada bajo condiciones socioecológicas y climatológicas diferentes ya que la vivienda experimental estaba ubicada en el chaco salteño, de clima más seco que Salvador Mazza, en un rancho cuyas paredes - de ladrillos de adobe - hacían junta con el techo, el cual era de encañado, paja y barro, es decir, más cerrado que las viviendas características de "El Chorro", por lo que resultaba más propicio para alcanzar y sostener la temperatura deseada. En este estudio, también se realizó el trabajo de campo durante el período de máxima actividad de los triatominos, ya que la temperatura ambiente en S. Mazza, como ya se dijo, tiene una media de $28,5^{\circ} \mathrm{C}$ entre diciembre y marzo, con máximas de hasta $48,8^{\circ} \mathrm{C}$.

Respecto de los insectos capturados vivos en la vivienda de adiestramiento, el adulto hallado en el suelo debajo de un tronco lo fue en razón de que no se aplicó el protocolo correctamente, ya que este indica claramente cómo debe prepararse el suelo de la habitación previo al tratamiento térmico ya que, justamente, la dispersión de las brasas es sustancial para lograr la destrucción de huevos y ninfas pequeñas ocultos en el polvillo del suelo. También lo es para destruir insectos que, huyendo de la mayor temperatura que se registra en lo alto de la construcción, descienden por las paredes donde quedan atrapados entre "dos fuegos" y mueren.

Los ejemplares hallados en el suelo 24 horas después por la dueña de casa eran inviables y todos habían muerto un día después. Sí debe aceptarse como válida la captura de una ninfa viva tras de un terrón que, además de existir la posibilidad de que haya sobrevivido al calentamiento, puede que reingresara luego de fugarse del mismo (aunque este hecho no fue detectado durante el procedimiento) o que se introdujera desde el gallinero colindante durante la noche, lugar altamente infestado.

Respecto de los resultados obtenidos en la vivienda seleccionada para la investigación, la captura del ejemplar adulto 24 horas después, sobre un tirante del techo, inclina a pensar en el reingreso de un animal previamente albergado en su interior. En cuanto a los dos ejemplares adultos marcados, cabe señalar que el espesor de la pared de adobe de esa vivienda es de unos $23 \mathrm{~cm}$, por lo que puede deducirse que la temperatura en el interior de la grieta alcanzó los $65^{\circ} \mathrm{C}$, toda vez que ese tipo de daño es observable en laboratorio a esa temperatura.

Con relación al efecto ovicida del calor en el lote expuesto, fue idéntico al observado en condiciones de laboratorio (100\%).

Los moradores de la vivienda, previo a la elevación térmica, pernoctaban a la intempe- 
rie, atemorizados por la abundancia de triatomas (se trata de familias que han recibido educación sanitaria durante 25 años, donde la prevención de la transmisión vectorial de la Enfermedad de Chagas forma parte de los ejes temáticos que desarrollan sistemáticamente los Agentes Sanitarios del Programa de APS, vigente allí desde 1978). Tras el procedimiento, la familia decidió utilizar el dormitorio esa misma noche, por lo que se la instruyó para que capturen y entreguen a su Agente Sanitario los vectores que avistaran, hecho que no se produjo entre las 48 horas y los 90 días posteriores, lo que coincide con la negatividad de las evaluaciones hechas en ese lapso.

\section{Conclusiones}

La elevación artificial de la temperatura del aire estanco en las habitaciones colonizadas por $T$. infestans, resultó ser un método de control de eficacia aceptable, seguro en manos de personal idóneo, técnicamente sencillo y aceptado por las familias participantes, lo cual lo posiciona como una alternativa a la utilización de órgano fosforados (Fenitrothión), decidida por la Coordinación Nacional de Control de Vectores - responsable de la lucha antivectorial en la Provincia de Salta - ante la emergencia sanitaria ocasionada por la reinfestación masiva de viviendas con vinchucas resistentes a piretroides, documentada en Salvador Mazza, Provincia de Salta, Argentina.

\section{Misceláneas}

\section{Alternativas tecnológicas}

Como desprendimientos de esta experiencia con Aerotermia, han surgido diversas alternativas de mejoras tecnológicas, tanto para la utili-

\section{Resumen}

Se exploraron los efectos de la elevación artificial de la temperatura del aire sobre las formas embrionarias, ninfales y adultas de Triatoma infestans resistentes a Deltametrina. En laboratorio, se expuso a temperaturas crecientes del aire la progenie completa de $\mathrm{T}$. infestans, para determinar viabilidad de huevos y supervivencia en ninfas y adultos; sobre el terreno, la experimentación se llevó a cabo en una vivienda tipo rancho previamente evaluada como colonizada. Durante la fase de laboratorio se comprobó la inviabilidad del $100 \%$ de los huevos y la mortalidad del $100 \%$ de las ninfas y adultos expuestos a temperaturas del aire es- zación de aire estanco, a lo que hemos denominado recientemente Aerotermia Estática, o en movimiento, Aerotermia Dinámica.

En el primer caso, consiste en la utilización de un cobertor de film plástico (130 micras de espesor) que cubre la totalidad de la vivienda para evitar las fugas de calor cuando es de construcción tan abierta que dificulta la utilización de materiales de hermetización o cuando por su construcción o deterioro, existen múltiples grietas abocadas al exterior. También lo hemos encontrado muy útil en días de bajas temperaturas.

En AT Dinámica, experimentamos recientemente con un equipo de manufactura artesanal, los efectos de la inyección de aire sobrecalentado a baja presión, directamente introducido con boquillas en los refugios de T. infestans intradomiciliarios, con excelentes resultados preliminares. Este dispositivo portátil, que pesa $2,5 \mathrm{~kg}$ y también utiliza como fuente calórica carbón vegetal $(1,5 \mathrm{~kg})$ se conecta a un compresor manual de fumigación (de los utilizados por el Programa Nacional de Control de Vectores) para la inyección del aire caliente. Aplicando el mismo principio, es teóricamente factible la adaptación de las moto-rociadoras utilizadas en la aplicación de insecticidas, para inyectar el aire sobrecalentado a partir del calor emitido por el motor.

Finalmente, hemos experimentado en el terreno, la asociación del método descrito en este trabajo con la utilización de un calefactor industrial portátil que emite $35.000 \mathrm{~K} / \mathrm{Cal} / \mathrm{hora}$, lo cual permite acortar drásticamente el procedimiento, al producirse una elevación brusca de la temperatura del aire hasta poco más de $100^{\circ} \mathrm{C}$, lo que, sumado a la gran turbulencia que genera, fuerza su circulación por los refugios de los insectos hasta calcinarlos. El bajo costo adquisitivo y operativo de este equipo y su sencillo y seguro manejo, lo posicionan como una alternativa muy ventajosa. tanco de entre $45^{\circ} \mathrm{C}$ y $55^{\circ} \mathrm{C}$, mientras que en la fase de terreno se logró reproducir el efecto ovicida del calor como así también la negativización de las evaluaciones entomológicas de la vivienda de experimentación, llevadas a cabo a las 72 horas, a los 30, 60 y 90 días. La elevación artificial de la temperatura del aire estanco en las habitaciones resultó ser un método de control de eficacia aceptable, inocuo y técnicamente sencillo.

Enfermedad de Chagas; Triatoma; Control Vectorial; Resistencia e Insecticida 


\section{Colaboradores}

Si bien todas y cada una de las actividades protocolizadas desarrolladas durante la investigación fueron compartidas por los cuatro integrantes del equipo, los borradores fueron discutidos mayormente entre los tres primeros autores, aunque por razones de distancia, el intercambio de impresiones para la redacción final se realizó por vía telefónica y e-mail. La redacción final del artículo enviado a CSP estuvo a cargo de A. G. Gentile.

\section{Referencias}

1. Barrios S, Vassena CV, González-Audino P, Picollo MI, Zerba EN. Caracterización de la resistencia a Deltametrina en Triatoma infestans de la Provincia de Salta. Buenos Aires: Centro de Investigaciones de Plagas e Insecticidas; 2001.

2. Centro de Investigaciones de Plagas e Insecticidas. Monitoreo de la resistencia a insecticidas en Triatoma infestans del República Argentina. Buenos Aires: Centro de Investigaciones de Plagas e Insecticidas; 1997/1998.

3. Ministerio de Salud Pública de Salta. Programa de Atención Primaria de la Salud - A.O. VII. Salvador Mazza (1985-2003) Informes Generales de Ronda Sanitaria. Salta: Ministerio de Salud Pública de Salta; s.d.

4. Ministerio de Salud de la Nación. Proyecto de certificación de la interrupción de Chagas vectorial en la Provincia de Salta. Salta: Programa Nacional de Chagas, Coordinación Nacional de Control de Vectores, Ministerio de Salud Pública de la Provincia de Salta; 2001.

5. Arnold CY. Maximum-minimum temperatures as a basic for computing heat units. Proc Am Soc Hort Sci 1960; 76:682-92.

6. Baskerville GL, Emin P. Rapid estimation of heat accumulation from maximum and minimum temperatures. Ecology 1969; 50:514-7.

\section{Agradecimientos}

A las autoridades del Ministerio de Salud Pública de la Provincia de Salta; al Dr. Alejandro Tapia, Gerente General del Área Operativa VII - Salvador Mazza; al personal del Programa Nacional de Chagas con asiento en Salvador Mazza. A las familias que ofrecieron sus viviendas, por su confianza, paciencia y colaboración. Al Dr. Enrique Vázquez de OPS Argentina por aceptarnos revisar el trabajo.

7. Belehradek J. Temperature and living matter. Berlin: Verlag Gebrüder Brontraeger; 1935. (Protoplasma Monogr. 8).

8. Briere JF, Pracros P, Le Roux AY, Pierre JS. A novel model of temperature-dependent development for arthropods. Environ Entomol 1999; 28: 22-9.

9. Marco V. Entomología aplicada III: modelización de la tasa de desarrollo de insectos en función de la temperatura. Aplicación al Manejo Integrado de Plagas mediante el método de grados-día. Bol S.E.A. 2001; 28:147-50.

10. Silveira AC, Arias AR, Segura E, Guillén G, Russomando G, Schenone H, et al. El control de la enfermedad de Chagas en los países del Cono Sur de América. Historia de una iniciativa internacional. 1991/2001. Washington DC: Organización Panamericana de la Salud; 2002.

11. Wagner TL, Wu H, Sharpe PJH, Schoofield RM, Coulson RN. Modeling insect development rates: a literature review and application of a biophysical model. Ann Entomol Soc Am 1984; 77:208-25.

Recibido el 15/Ago/2003

Versión final presentada el 02/Mar/2004

Aprobado el 12/Mar/2004 